\title{
Long-term continuously monocropped peanut significantly changed the abundance and composition of soil bacterial communities
}

\author{
Mingna Chen ${ }^{1,2}$, Hu Liu ${ }^{1}$, Shanlin Yu ${ }^{2}$, Mian Wang ${ }^{2}$, Lijuan Pan ${ }^{2}$, Na Chen ${ }^{2}$, Tong Wang ${ }^{2}$, Xiaoyuan Chi ${ }^{\text {Corresp., }}{ }^{2}$ \\ Binghai Du ${ }^{\text {Corresp. } 1}$ \\ ${ }^{1}$ College of Life Sciences, Shandong Key Laboratory of Agricultural Microbiology, National Engineering Laboratory for Efficient Utilization of Soil and \\ Fertilizer Resources, Shandong Agricultural University, Taian, China \\ 2 Shandong Peanut Research Institute, Qingdao, China \\ Corresponding Authors: Xiaoyuan Chi, Binghai Du \\ Email address: chi000@126.com, dubhai@126.com
}

Soil sickness is the progressive loss of soil quality due to continuous monocropping. The bacterial populations are critical to sustaining agroecosystems, but their responses to long-term peanut monocropping have not been determined. In this study, based on a previously constructed gradient of continuous monocropped plots, we tracked the detailed feedback responses of soil bacteria to short- and long-term continuous monocropping of four different peanut varieties using high-throughput sequencing techniques. The analyses showed that soil samples from 1- and 2-year monocropped plots were grouped into one class, and samples from the 11- and 12-year plots were grouped into another. Long-term consecutive monocropping could lead to a general loss in bacterial diversity and remarkable changes in bacterial abundance and composition. At the genera level, the dominant genus Bacillus changed in average abundance from $1.49 \%$ in short-term monocropping libraries to $2.96 \%$ in the long-term libraries. The dominant species Bacillus aryabhattai and Bacillus funiculus and the relatively abundant species Bacillus luciferensis and Bacillus decolorationis all showed increased abundance with long-term monocropping. Additionally, several other taxa at the genus and species level also presented increased abundance with long-term peanut monocropping; however, several taxa showed decreased abundance. Comparing analyses of predicted bacterial community functions showed significant changes at different KEGG pathway levels with long-term peanut monocropping. Combined with our previous study, this study indicated that bacterial communities were obviously influenced by the monocropping period, but less influenced by peanut variety and growth stage. Some bacterial taxa with increased abundance have functions of promoting plant growth or degrading potential soil allelochemicals, and should be closely related with soil remediation and may have potential application to relieve peanut soil sickness. A decrease in diversity and abundance of bacterial communities, Peer] reviewing PDF | (2019:12:43788:2:0:NEW 10 Mar 2020) 
especially beneficial communities, and simplification of bacterial community function with long-term peanut monocropping could be the main cause of peanut soil sickness. 
1 Long-Term Continuously Monocropped Peanut Significantly Changed the Abundance and Composition of Soil Bacterial Communities

3 Mingna Chen ${ }^{1,2}, \mathrm{Hu} \mathrm{Liu}^{1}$, ShanLin $\mathrm{Yu}^{2}$, Mian Wang², LiJuan $\mathrm{Pan}^{2}$, Na Chen², Tong Wang ${ }^{2}$, Xiaoyuan Chi2*, 4 Binghai $\mathrm{Du}^{1 *}$

5 1. College of Life Sciences, Shandong Key Laboratory of Agricultural Microbiology, National Engineering

6 Laboratory for Efficient Utilization of Soil and Fertilizer Resources, Shandong Agricultural University, Tai'an,

7 China

8 2. Shandong Peanut Research Institute, Qingdao, China

9 Corresponding authors:

\section{Prof. Binghai Du}

Shandong Agricultural University

No. 61 Daizong Road, Taishan District, 271018, Tai'an, China

E-mail: dubhai@126.com

Phone: $86-538-8242908$

Fax: 86-538-8242908

\section{Prof. Xiaoyuan Chi}

Shandong Peanut Research Institute

No. 126 FuShan Road, Licang District, 266100, Qingdao, China

E-mail: chi000@126.com

Phone: 86-532-87626672

Fax: 86-532-87626832 


\section{ABSTRACT}

25 Soil sickness is the progressive loss of soil quality due to continuous monocropping. The

bacterial populations are critical to sustaining agroecosystems, but their responses to long-term

peanut monocropping have not been determined. In this study, based on a previously constructed gradient of continuous monocropped plots, we tracked the detailed feedback responses of soil bacteria to short- and long-term continuous monocropping of four different peanut varieties using high-throughput sequencing techniques. The analyses showed that soil samples from 1and 2-year monocropped plots were grouped into one class, and samples from the 11- and 12year plots were grouped into another. Long-term consecutive monocropping could lead to a general loss in bacterial diversity and remarkable changes in bacterial abundance and composition. At the genera level, the dominant genus Bacillus changed in average abundance from $1.49 \%$ in short-term monocropping libraries to $2.96 \%$ in the long-term libraries. The dominant species Bacillus aryabhattai and Bacillus funiculus and the relatively abundant species Bacillus luciferensis and Bacillus decolorationis all showed increased abundance with long-term monocropping. Additionally, several other taxa at the genus and species level also presented increased abundance with long-term peanut monocropping; however, several taxa showed decreased abundance. Comparing analyses of predicted bacterial community functions showed

41 significant changes at different KEGG pathway levels with long-term peanut monocropping.

42 Combined with our previous study, this study indicated that bacterial communities were obviously influenced by the monocropping period, but less influenced by peanut variety and growth stage. Some bacterial taxa with increased abundance have functions of promoting plant 
45

46

47

48

growth or degrading potential soil allelochemicals, and should be closely related with soil remediation and may have potential application to relieve peanut soil sickness. A decrease in diversity and abundance of bacterial communities, especially beneficial communities, and simplification of bacterial community function with long-term peanut monocropping could be the main cause of peanut soil sickness.

\section{INTRODUCTION}

Soil sickness is the progressive loss of soil quality due to continuous monocropping and results in the reduction of crop yield and quality, as well as a prevalence of soil-borne diseases (Huang et al., 2013; Van der Putten et al., 2013). It is a major problem in agriculture ecosystems all over the world, and has been reported for many types of crops, including food (e.g., rice, wheat, corn, soybean, and peanut), economic (e.g., sugarcane and tobacco), vegetable (e.g., cucumber and eggplant), and medicinal crops (e.g., Rehmannia, ginseng, and Angelica) (Liu et al., 2012; Gentry et al., 2013; Huang et al., 2013; Wu et al., 2015). Monocropping is considered unsustainable in agricultural systems; however, modern agricultural practices are often characterized by monocropping (Cook, 2006).

Based on previous reports, there are four main factors contributing to soil sickness: disorder in physicochemical soil properties, production, and accumulation of autotoxins, imbalance of soil microbial communities and change in soil enzyme activity (Huang et al., 2013; Zhou et al.,2018). Soil microorganisms that are critical to many soil biological, chemical, and physical processes such as soil structure formation, mineral nutrition cycling, organic matter turnover and toxin accumulation or removal, are considered to be key drivers of terrestrial ecosystems (Bever et al., 
66 2012; Blagodatskaya and Kuzyakov, 2013). Alteration of soil microbial communities can change

67 the function performed by the communities and then feedback on plant growth and health (Bever

68 et al., 2012; Zhou et al., 2017). In addition, the soil microbial community can serve as a sensitive

69 bioindicator of soil health due to its quick response to environmental changes and close

70 relationship with soil conditions and land management (Sharma et al., 2010). Consequently,

71 understanding how soil microbial communities are affected by continuous monocropping is

72 necessary to provide insights into soil sickness.

73 Bacterial populations are most abundant and diverse in soil, and the interactions between

soil, bacteria, and plants in root-related environments play key roles in soil fertility, sustainability,

and plant quality (Chaparro et al., 2012). Many bacterial taxa have been identified as biocontrol

agents against soil-borne pathogens and play key roles in promoting plant growth, and some soil

bacteria also have been reported as plant pathogens (Compant et al., 2010; Santoyo et al., 2012;

Buttimer et al., 2017). Increasing evidence indicates that the soil bacterial communities can be

shaped by plants through secretion of root exudates (Doornbos et al., 2012). Modifications in

soil microbe populations induced by peanut (Arachis hypogaea L.) root exudates, rather than

direct allelopathy, could contribute to peanut soil sickness (Li et al., 2014a). Additionally, recent

studies suggested that accumulations of microbial pathogens at the expense of plant-beneficial

microorganisms in the soil are likely explanations for yield declines as a consequence of consecutive monocropping (Chen et al., 2012; Li et al., 2014b; Xiong et al., 2015). Therefore, 
87 Peanut, an important oil and economic crop worldwide, is very adaptable to climatic

88 conditions and grows in tropical, subtropical, and warm temperate climate regions across the world. Due to limitations of arable land and requirements for developing regional agroindustrialization, large-scale monocropping of peanut is common in China (Chen et al., 2016). Research indicates that consecutive peanut monocropping has caused a decline in yield and quality and increases in disease pressures (Wang et al., 2005). Early studies showed that soil diversity and abundance of bacterial communities changed with continuous peanut monocropping according to phospholipid fatty acid (PLFA), denaturing gradient gel electrophoresis and library analyses (Li et al., 2012; Chen et al., 2014; Liu et al., 2015). Our earlier study also indicated that the balance of soil bacterial communities was disturbed during three years of continuous monocropping (Chen et al., 2014). However, the specific characteristics of the soil bacterial community and the changes of soil bacterial structure and composition in response to long-term peanut monocropping are unclear.

In this study, based on a gradient that we previously constructed of continuously monocropping in a peanut field, we analyzed and compared responses of root soil bacterial communities of four peanut varieties to monocropping for $1,2,11$, and 12 years, using high-throughput sequencing techniques. This study aims to investigate bacterial community dynamics succession under longterm peanut monocropping based on monocropping gradient experiment plots with a consistent background. The aims of this study were to (i) determine the change characteristics of the soil bacterial community and the influences of peanut varieties on the dynamics of the bacterial community, under long-term continuous monocropping of peanut, and (ii) identify the key 
108

109

110

111

112

113

114

115

116

117

118

119

120

121

122

123

124

125

126

127

bacteria taxa related to peanut soil sickness.

\section{MATERIALS AND METHODS}

\section{Field experiment and soil sampling}

The field experimental site was set up in Laixi experimental farm at the Shandong Peanut

Research Institute, Qingdao, China $\left(36^{\circ} 50^{\prime} \mathrm{N}, 120^{\circ} 31^{\prime} \mathrm{E}\right)$. A gradient of consecutive monocropped peanut experiment plots that we previously developed was used. Independent pools were applied, with the size of each pool being $4 \mathrm{~m}$ long, $1.5 \mathrm{~m}$ wide, and $1 \mathrm{~m} \mathrm{high}$. We collected soil from the plow layer of the cultivated land that had previously been planted with a wheat-maize rotation, thoroughly mixed, and then added to the pools. In order to construct the gradient of continuous monocropping plots, sweet potatoes were rotated with peanut in some plots during the experiment. Peanuts were planted in May each year and harvested in October. All field management, including the planting pattern and the use of water and fertilizer were consistent among different pools. After harvest, the plots lay fallow until the next planting season.

The distance between rows was $16.5 \mathrm{~cm}$ and the ridge width was $70 \mathrm{~cm}$, with two peanut seeds per hole. Before planting, about $300 \mathrm{~kg} \mathrm{ha}^{-1}$ of urea, $750 \mathrm{~kg} \mathrm{ha}^{-1}$ of calcium magnesium phosphate, and $225 \mathrm{~kg} \mathrm{ha}^{-1}$ of potassium chlorate were applied as fertilizer. The weeds were controlled using glyphosate ( $41 \%$ active ingredient in $3 \mathrm{~L} \mathrm{ha}^{-1}$ ) before planting. Plastic film mulching was applied during cultivation. In addition, to minimize the influence of other factors including variety on the measured indexes, the planting position of the same peanut variety was fixed during the continuous cropping period. The longest-running pools have now been 
128

129

130

131

132

133

134

135

136

137

138

139

continuously monocropped with peanuts for 16 years.

According to our previous study (Jiao et al., 2015), we selected four peanut varieties with distinct responses to monocropping to analyze their soil bacterial community structure in plots monocropped for $1,2,11$, and 12 years. The four varieties included two large fruit varieties, Huayu 917 and Huayu 50, and two small fruit varieties, Huayu 26 and Huayu 20. Based on previous testing of their yield-related indexes, Huayu 20 was the most sensitive to long-term monocropping, Huayu 917 was intermediate, and Huayu 26 and Huayu 50 were tolerant. There were three replicates for each treatment. The bulk soils were collected at the full-bloom stage and five randomly selected replicate test plants were used for each sample. The soils around individual plants were sampled using a soil probe $(1.5 \mathrm{~cm}$ diameter $)$ at $5-10 \mathrm{~cm}$ soil depth, and at distances of $3-5,8-10$, and $13-15 \mathrm{~cm}$ from the main root. The root zone soils were then mixed together. These distances were chosen because the roots at this depth and these distances were relatively abundant and the soil microbial community around the peanut root system could be well characterized (Chen et al., 2012). Physico-chemical properties of $\mathrm{pH}$, organic matter, and available nitrogen $(\mathrm{N})$, phosphorus $(\mathrm{P})$, and potassium $(\mathrm{K})$ in the soil samples were determined using routine methods (Lu 1999). Samples Y1, Y2, Y11, and Y12 were for monocropping of 1, 2, 11, and 12 years, respectively; H20, H26, H50, and H917 were samples for four peanut varieties with distinct responses to monocropping.

\section{DNA extraction, PCR, and high-throughput sequencing}

For each soil sample, total DNA was extracted with the Power Soil DNA Isolation Kit (MoBio Laboratories Inc., Carlsbad, CA, USA) following the manufacturer's instructions. The concentration and purity of the DNA was checked by electrophoresis on $1.0 \%(\mathrm{w} / \mathrm{v})$ agarose gels. Soil bacterial communities were analyzed with amplicon sequencing on an Illumina HiSeq platform. Two primers, 515F (5'-GTGCCAGCMGCCGCGGTAA-3') and 907R (5'- 
152 CCGTCAATTCCTTTGAGTTT-3'), for the V4-V5 region of the 16S rRNA gene were applied.

153 Both the forward and reverse primers had a 6-bp barcode unique to each sample. Each soil

154 sample was independently amplified in triplicate, and the triplicate PCR reaction products for

155 each sample were pooled and then all products were purified using a GeneJET ${ }^{\mathrm{TM}}$ gel extraction

156 kit (Thermo Scientific, Waltham, USA). The purified amplicons from each sample were pooled

157 in equimolar concentrations and the mixture was then sequenced on an Illumina HiSeq platform

158 at Novogene Co. Ltd, Beijing, China.

159 Sequencing data processing and statistical analysis

160 The paired-end reads from the original DNA fragments were merged with FLASH software

161 (Edgar, 2013). The QIIME 1.7.0 software package was used to quality-filter and process the raw

162 sequencing reads (Caporaso et al., 2010). The UCLUST method was used to delineate the

163 operational taxonomic units (OTUs) at a threshold of 97\% identity (Caporaso et al., 2010). A

164 representative sequence for each OTU was taxonomically classified using the Silva Database

165 (https://www.arb-silva.de/) based on Mothur algorithm (Quast et al., 2013). The OTU abundance

166 data were normalized using a standard sequence number corresponding to the sample with the

167 least sequences. The output normalized data were used for the subsequent analyses.

168 To assess the bacterial alpha-diversity of each sample, analyses including rarefaction curves,

169 Shannon index, and Chao1 index were performed using QIIME (Version 1.7.0) and displayed

170 with $\mathrm{R}$ software (Version 2.15.3). Beta diversity analyses were used to estimate sample

171 differences in bacterial community compositions. In order to analyze the influence of peanut 
172 monocropping on soil bacterial community composition, heatmap analysis was performed with $\mathrm{R}$

173 software. Cluster analysis was preceded by principal component analysis (PCA), which was

174 applied to reduce the dimensions of the original variables using the FactoMineR package and

175 ggplot2 package in $\mathrm{R}$ software (Version 2.15.3). Principal coordinate analysis (PCoA) was

176 performed to obtain principal coordinates and visualize the complex multidimensional data. The

177 PCoA analysis was performed using the WGCNA, stat, and ggplot2 packages in R software

178 (Version 2.15.3). Unweighted pair-group method with arithmetic means (UPGMA) clustering

179 was performed as a type of hierarchical clustering method to interpret the distance matrix using

180 average linkage and was conducted using QIIME software (Version 1.7.0). Taxa with

181 significantly diverse abundance with long-term monocropping at different levels were further

182 investigated using the Metastats method and t-test using R software (Version 2.15.3). Tax4Fun

183 functional prediction was achieved by the nearest neighbor method based on the minimum 16S

184 rRNA sequence similarity. The data set was deposited in the NCBI-Sequence Read Archive with

185 the submission Accession Number PRJNA559575.

186 RESULTS

187 Physico-chemical properties of soil

188 Physico-chemical properties of $\mathrm{pH}$, organic matter, and available $\mathrm{N}, \mathrm{P}$, and $\mathrm{K}$ in the peanut 189 monocropping soil were tested and analyzed (Table S1). The soil pHs in samples from 2-year 190 monocropped plots (8.30-8.59) were slightly higher than those from 1-year monocropped plots 191 (7.20-8.05), but were obviously lower in samples from 11- and 12-year monocropped plots 
192 (6.46-6.92). The contents of available $\mathrm{P}$ were lower in long-term $\left(15.12-21.12 \mathrm{mg} \mathrm{kg}^{-1}\right)$ than in

193 short-term monocropping samples (39.37-48.58 $\left.\mathrm{mg} \mathrm{kg}^{-1}\right)$. The contents of available $\mathrm{K}$ were also

194 lower in long-term $\left(59.98-86.13 \mathrm{mg} \mathrm{kg}^{-1}\right)$ than in short-term monocropping samples (99.42-

$195120.67 \mathrm{mg} \mathrm{kg}^{-1}$ ). However, contents of organic matter and available $\mathrm{N}$ showed no obvious 196 changes with long-term peanut monocropping.

197 Characteristics of sequencing data

198

199

200

201

202

203

204

205

206

207

208

209

210

211

In order to compare the soil bacterial community structure and composition of the four peanut varieties to short- and long-term monocropping periods, a total of $4816 \mathrm{~S}$ rRNA gene libraries were analyzed using high-throughput sequencing. The sampling variables, including four peanut varieties and four monocropping periods, are shown in Table 1. A total of 2,541,421 quality-filtered sequences obtained from the 48 samples, ranging within 37,605-68,117 with an average length of $373 \mathrm{bp}$, resulted in identification of a total of 9028 OTUs applying a 3\% sequence dissimilarity cutoff. After data normalization, 1,805,040 quality-filtered sequences affiliated with 8856 OTUs were obtained. The bacterial complexity of the 48 samples was estimated on the basis of alpha-diversity (OTU number, Chaol index, and Shannon index) and showed relatively higher bacterial diversity in samples from the 1- and 2-year compared to the 11- and 12-year monocropped plots (Table 1). Rarefaction curves (Figure S1) and indices of richness and diversity including all samples' Chaol and Shannon indexes (Table 1) tended to approach a saturation plateau, indicating that the majority of bacterial diversity was recovered by the surveying effort. 


\section{Bacterial community structure and composition}

In total, $99.73 \%$ of the identified 8856 OTUs were related to bacteria, and the other 24

OTUs accounting for $0.15 \%$ of the total quality-filtered sequences were affiliated with Archaea.

There were 41 bacteria phyla detected across all samples and $0.73 \%$ of bacterial sequences were

unclassified at the phylum level. The top 10 phyla accounted for $95.49 \%$ of the total sequences

and $79.67 \%$ of the total OTUs. The dominant phyla across all samples were Proteobacteria,

Actinobacteria, Acidobacteria, and Chloroflexi, accounting for 30.71, 21.59, 18.04, and 7.69\% of

the total sequences, respectively, and $26.61,7.43,6.49$, and $10.77 \%$ of the total 8856 OTUs,

respectively. The phyla Gemmatimonadetes, Firmicutes, Planctomycetes, Nitrospirae,

Bacteroidetes, and Thermomicrobia were relatively abundant and diverse, accounting for 4.66,

$3.75,3.13,2.96,1.62$, and $1.36 \%$ of the total sequences, respectively, and $3.42,4.41,14.76,0.61$,

3.97, and $1.19 \%$ of the total OTUs, respectively (Figure S2).

At the class level, 92 bacteria taxa were identified. The dominant 19 taxa (relative

abundance $>1 \%$ ) accounted for $>88.43 \%$ of the sequences in each sample. The most abundant

taxa (relative abundance $>5 \%$ ) were Acidobacteria (16.19\%), Alphaproteobacteria (10.11\%),

Betaproteobacteria

$(9.48 \%)$

$(9.22 \%)$

Actinobacteria

$(7.31 \%)$

Deltaproteobacteria (5.77\%), and Gammaproteobacteria (5.29\%). The other relatively abundant

229 taxa included Gemmatimonadetes, Bacilli, Nitrospira, MB-A2-108, Planctomycetacia,

Acidimicrobiia, Anaerolineae, KD4-96, Holophagae, Thermomicrobia, Sphingobacteriia, and

TK10, each accounting for $1.05-4.66 \%$ of the total sequences (Figure 1A). 
At the genus level, 494 taxa were detected, accounting for $31.41 \%$ of the total sequences;

and the top 50 taxa accounted for $75.04 \%$ of the total identified sequences at the genus level. The dominant genera (relative abundance $>1 \%$ ) were Bacillus, Gaiella, Mizugakiibacter, and

Streptomyces, accounting for 2.22, 1.79, 1.35, and 1.01\% of the total sequences, respectively.

Relatively abundant genera were Sphingomonas (0.90\%), Haliangium (0.89\%), Gemmatimonas samples. Overall, the UPGMA tree showed that replicate samples were grouped together, and soil samples with similar monocropping time but different peanut varieties were clustered into a group (Figure 2). Both PCA and PCoA also showed obvious clustering of bacterial communities plots were grouped into one class, and samples from 11- and 12-year plots were grouped into another. However, the cluster distance was shorter among samples from 1- and 2-year than among samples from 11- and 12-year monocropping plots. These analyses indicated that showed more obvious changes under long-term monocropping. 
252 diverse at different levels between short- and long-term monocropping. At the phylum level, 253 among the 41 bacteria taxa identified, nine taxa showed an obvious decrease in abundance with 254 monocropping time and five taxa showed an obvious increase in abundance (Figure S4, Figure 4). 255 The average abundance of Acidobacteria accounted for 20.30 and $19.83 \%$ in the 1- and 2-year 256 libraries, respectively, and decreased to 15.74 and $16.28 \%$ in the $11-$ and 12-year libraries. The 257 phyla Planctomycetes, Nitrospirae, and Bacteroidetes were relatively abundant in the 1- and 2258 year libraries, accounting for $3.12,2.96$, and $1.62 \%$ of the total sequences, respectively, and 259 correspondingly decreased in the 11- and 12-year libraries by 20.23, 17.47, and $27.81 \%$. 260 Additionally, the taxa Armatimonadetes, Latescibacteria, JL-ETNP-Z39, Thermotogae, and 261 Caldiserica, for which relative abundances were $<1 \%$, also had clear decreasing tendencies. In 262 general, decreases in their abundance were accompanied by decreases in their diversity (Table 263 S2). In contrast, the abundance of some taxa increased with monocropping time. The average 264 abundance of Gemmatimonadetes, Firmicutes, and Elusimicrobia accounted for 3.91, 2.65, and $0.16 \%$ in the short-term monocropping libraries, respectively, and correspondingly increased to 5.40, 4.84, and $0.21 \%$ for the long-term. The other taxa with increasing abundance all had relatively low abundance, accounting for $0.01-0.04 \%$ of the total sequences, and included 268 Parcubacteria and Chlamydiae. The diversity of the three relatively abundant taxa 269 (Gemmatimonadetes, Firmicutes, and Elusimicrobia) did not show obvious changes with monocropping time, but that of the other low-abundance taxa tended to increase with monocropping time (Table S2). 
273 provide better phylogenetic resolution than at higher taxonomic levels (Ho et al., 2016). At the 274 genera level, the top 50 most-abundant taxa accounted for $75.04 \%$ of the total identified 275 sequences that were affiliated with 494 taxa. The abundance distributions of the top 50 genera in 276 each sample were presented in a heatmap (Figure 5). Some taxa showed obvious changes with 277 monocropping time (Figures S5 and S6). The dominant genera Bacillus (2.22\%) and 278 Mizugakiibacter (1.35\%) increased their average abundance from 1.49 and $0.67 \%$, respectively, 279 in the 1- and 2-year libraries to 2.96 and $2.02 \%$ in the 11- and 12-year libraries. However, the number of OTUs affiliated with the genera Bacillus (13-14 OTUs) and Mizugakiibacter (four OTUs) in each sample was relatively low and changed little. Seven genera (Gemmatimonas, Marmoricola, Candidatus Solibacter, Tumebacillus, Jatrophihabitans, Sporosarcina, and Pseudolabrys) accounted for $0.17-0.80 \%$ of the total sequences, and increased their average abundance by $>90 \%$. The genera Nocardioides, Solirubrobacter, and Reyranella accounting for $0.21-0.74 \%$ of the total sequences also showed increased abundance with monocropping time, with increase rates of $26.44-57.20 \%$. Some taxa showed an opposite trend, with abundance decreasing with monocropping time. They were Steroidobacter, Candidatus Entotheonella,

Nitrosospira, Pirellula, Piscinibacter, Ramlibacter, Lysobacter, Skermanella, and Planctomyces, which accounted for $0.20-0.68 \%$ of the total sequences, and the abundance decrease rate ranged within 12.86-51.13\%. Overall, abundance of these genera began to decline in the 2-year monocropping libraries, and decreased more for the long-term libraries. 
294 monocropping time (Figure S7, Figure 6). Among the top 10 most-abundant species, Bacillus

295 aryabhattai and Bacillus funiculus accounting for 0.58 and $0.42 \%$ of the total sequences,

296 respectively, showed increased abundance in long- compared to short-term monocropping

297 libraries. The increase rate of these two taxa reached 116.23 and $177.70 \%$, respectively. The

298 relatively abundant taxa of Bacillus luciferensis, Tumebacillus ginsengisoli, Bacillus

299 decolorationis, and Streptacidiphilus luteoalbus, accounting for $0.01-0.10 \%$ of the total

300 sequences, increased their relative abundances by $>118 \%$. The taxa Paenibacillus alginolyticus

301 and Streptosporangium violaceochromogenes also presented increased abundance with increase

302 rates ranging within $52.07-84.49 \%$, but the change trends differed among peanut varieties. The

303 species Paenibacillus alginolyticus and Streptosporangium violaceochromogenes showed no

304 obvious changes in abundance in Huayu 917 and Huayu 50 libraries, respectively. In contrast to

305 the above taxa, Chitinophaga ginsengihumi, Lysobacter yangpyeongensis, and Phyllobacterium

306 myrsinacearum, accounting for $0.03-0.05 \%$ of the total sequences, showed obvious decreases in

307 abundance and the abundance decrease rate ranged within $32.57-61.59 \%$.

308 Prediction of bacteria community functions

The predominant category of the predicted functional genes was affiliated with metabolism

$310(48.71 \%)$, followed by genetic information $(21.03 \%)$, environmental information processing

$311(12.89 \%)$, and cellular processes (7.62\%) (Figure S8). The PCA was used to cluster the predicted

312 functional pathway within soil samples at three KEGG levels. The analyses at KEGG levels 2

and 3 and KEGG ortholog $(\mathrm{KO})$ level all showed similar clusters to that of bacterial community 
314 structure (Figure 7, Figure S9). Samples from 1- and 2-year monocropping plots were grouped

315 into one class, and samples from 11- and 12-year plots were grouped into another.

316 Bacterial community functions also presented significant changes at different pathway

317 levels across long-term monocropping. For KEGG pathways at KEGG level 2 that are involved

318 in carbohydrate metabolism, endocrine system, excretory system, nucleotide metabolism,

319 transport and catabolism, transcription, biosynthesis of other secondary metabolites, and aging

320 showed decreasing trends with long-term monocropping, but pathways affiliated with membrane

321 transport and cellular community prokaryotes showed increased abundance (Table S3). There

322 were 390 KEGG pathways at KEGG level 3 predicted in our libraries. The abundant pathways

$323(>1.0 \%)$ that presented obvious changes in abundance with monocropping period were further

324 examined. The pathways involved in DNA repair and recombination proteins, purine metabolism,

325 transfer RNA biogenesis, exosome, amino acid related enzymes, pyrimidine metabolism,

326 mitochondrial biogenesis, ribosome, oxidative phosphorylation, carbon fixation pathways in

327 prokaryotes, aminoacyl tRNA biosynthesis as well as alanine, aspartate, and glutamate

328 metabolism showed decreased abundance in long- compared to short-term monocropping

329 samples. In contrast, pathways involved in ribosome biogenesis, messenger RNA biogenesis,

330 RNA degradation, quorum sensing, glyoxylate and dicarboxylate metabolism, and lipid

331 biosynthesis proteins were enriched in long-term monocropping samples (Table S4).

\section{DISCUSSION}

333 It has been demonstrated that soil microorganisms play influential roles in the productivity 334 and sustainability of agricultural systems (Van der Heijden and Wagg, 2013; Vukicevich et al. 
335 2016). 'Soil sickness' caused by continuous peanut monocropping could be closely related to the

336 dynamics of soil microbial communities. Our previous study using library analysis showed that

337 soil microbial community structure shifted during three years of continuous peanut

338 monocropping (Chen et al., 2012; Chen et al., 2014). Li et al. reported that consecutive peanut

339 monoculture changed communities of soil nematodes and fungi (Li et al., 2014b; Li et al., 2015).

340 However, the specific dynamic succession of the bacterial community, which is most diverse in

341 soil, under long-term peanut monocropping is not clear.

342 In the present study, we selected four peanut varieties with different monocropping

343 responses and recorded the detailed feedback responses of their root soil bacteria communities to

344 short- and long-term consecutive monocropping using high-throughput sequencing. Bacterial

345 richness and diversity were measured by OTU number and Chaol and Shannon indexes, as well

346 as rarefaction curves. They all indicated that the majority of bacterial diversity was covered by

347 the surveying effort and the diversity of the soil bacterial community generally declined with

348 long-term peanut monocropping.

349 Our previous study showed that bacterial community structure presented significant

350 dynamics during three years of peanut monocropping, but was less affected by peanut growth

351 stage (Chen et al. 2014). Li et al. (2014b) reported that fungal communities were significantly

352 selected by the monocropping period of peanut, but also were less affected by growth stage in

353 the red soil region of southern China. In the present study, the UPGMA, PCA, and PCoA

354 methods were used to cluster the bacterial communities within the 48 samples from short- and 
355 long-term monocropping soil. The analyses showed that all samples regardless of variety from 1356 and 2-year monocropping plots were grouped into one class, and samples from 11- and 12-year 357 plots were grouped into another. However, the cluster distance between samples from 1- and 2358 year plots was shorter than that between samples from 11- and 12-year plots. Additionally, soil $359 \mathrm{pH}$ and contents of available $\mathrm{P}$ and $\mathrm{K}$ in soil also obviously changed with long-term monocropping of peanut. Monocropping time had a strong influence on the microbial communities as well as physico-chemical properties, but peanut variety and growth stage had little impact. Additionally, the soil bacterial community had significantly greater dynamics under long- compared to short-term monocropping time.

Our previous study demonstrated that bacterial communities at different taxonomic levels showed obvious dynamics during three years of peanut monocropping (Chen et al. 2014). Most of the obviously changed taxa at the order level showed abundance and diversity declines with monocropping time and only several taxa showed increased abundance and diversity (Chen et al. 2014). A third cropping of Jerusalem artichoke also decreased bacterial alpha-diversity compared to 1-2 years of monocropping (Zhou et al., 2018). In this study, under long-term peanut monocropping, the alpha-diversity of the soil bacterial community generally decreased. At the phyla level, nine taxa that accounted for $0.01-18.04 \%$ of the total sequences showed obvious

372 decreases in abundance with monocropping time and generally their decreased abundance was

373 accompanied by a decrease in diversity. Five taxa, representing $0.01-4.66 \%$ of the total sequences, showed obvious increases in abundance: the three relatively abundant taxa showed no 
375 obvious change in diversity with monocropping time, but that of the other two less abundant taxa

376 showed increasing trends. It was suggested that simplification of bacterial communities is a

377 common phenomenon during monocropping; however, some taxa increased their abundance and

378 diversity, possibly due to their adaptability to a new microenvironment.

379 Soil microbe modifications could contribute to peanut soil sickness (Chen et al., 2014; Li et al., 2014b). Li et al. (2012) reported that bacteria proportion in total PLFA decreased from 67.4

to $53.0 \%$ in a peanut monocropping system, whereas the proportion of fungi increased from 16.9

to $32.8 \%$. Consecutive peanut monocropping resulted in the selection of pathogenic and beneficial fungi (Chen et al., 2012; Li et al., 2014b). Soil nematode abundance and functional composition also changed with continuous peanut monocropping ( $\mathrm{Li}$ et al., 2015). In our study, the bacteria community compositions and predicted functions at different levels all presented obvious changes with long-term peanut monocropping.

Some taxa that were identified at lower taxonomic levels, such as genus or species, showed significant changes in abundance with long-term peanut monocropping. At the genera level,

Steroidobacter, Candidatus Entotheonella, Nitrosospira, Pirellula, Piscinibacter, Ramlibacter,

Lysobacter, Skermanella, and Planctomyces showed decreased abundance with monocropping

time. At the species level, Chitinophaga ginsengihumi, Lysobacter yangpyeongensis, and

Phyllobacterium myrsinacearum also showed obvious decreases with long-term monocropping.

Several taxa found were beneficial to plant growth. The genus Nitrosospira is reportedly related

to nitrogen cycle progress (Mellbye et al., 2017) and Candidatus Entotheonella is related to 
396

397

398

399

400

401

402

403

404

405

406

407

408

409

410

411

412

413

414 416 415 2003). There are no reports concerning roles in plants of Bacillus luciferensis and Bacillus

myrsinacearum functions in nitrogen-fixing (Gonzalez-Bashan et al., 2000). Studies concerning

roles in plants of the other decreased taxa at the genus or species levels were not found. However, this also demonstrated a decrease in abundance of the beneficial bacteria community with longterm peanut monocropping.

In contrast, some taxa at the genus or species levels showed increased abundance with longterm peanut monocropping. The genus Bacillus was dominant $(2.22 \%)$ in our libraries, and increased their average abundance from $1.49 \%$ in 1 - and 2-year to $2.96 \%$ in the 11 - and 12 -year libraries. However, the number of OTUs affiliated with the Bacillus genus in each sample (13-14 OTUs) was relatively low and changed little. This may suggest that identified members of Bacillus had good adaptability to the soil environment under long-term peanut monocropping. Bacillus species are reported as the most common biocontrol agents and have important traits such as plant growth-promoting properties (Santoyo et al., 2012; Gomaa et al., 2012). The dominant species Bacillus aryabhattai and Bacillus funiculus accounting for $0.58 \%$ and $0.42 \%$ of the total sequences, respectively, and the relatively abundant Bacillus luciferensis and Bacillus decolorationis accounting for 0.10 and $0.02 \%$ of the total sequences, respectively, all showed increased abundance with long-term monocropping. It was reported that Bacillus aryabhattai could improve growth of soybean, wheat, and Xanthium italicum, and could also improve mobilization and biofortification of zinc (Lee et al., 2012; Ramesh et al., 2014). Bacillus funiculus is reportedly related to the degradation of sodium dodecyl sulfate (Ajithkumar et al., decolorationis. Additionally, several relatively abundant species, including Tumebacillus 
417 ginsengisoli, Streptacidiphilus luteoalbus, Paenibacillus alginolyticus, and Streptosporangium

418 violaceochromogenes also showed increased abundance with long-term peanut monocropping.

419 However, no function-related studies of them were found. At the genus level, the functions of

420 most of the increased taxa are unknown, but some studies claimed that Nocardioides species had

421 roles in degradation of deoxynivalenol, 2,4-dinitroanisole, and melamine and its hydroxy

422 derivatives (Ikunaga et al., 2011; Takagi et al., 2012; Fida et al., 2014). It was reported that

423 allelochemicals from root exudates or decomposants of crops could induce autotoxicity and were

424 closely related to soil sickness (Asaduzzaman et al., 2012; Huang et al., 2013). These

425 allelochemicals accumulated with monocropping period, but not to high levels (Yang et al., 2015;

426 Li et al., 2014a), possibly due to interactions between allelochemicals and soil microbes ( $L i$ et al.,

427 2014a; Wang et al., 2019). Based on the analyses, the increased bacterial taxa that could promote

428 plant growth or degrade potential soil allelochemicals should be closely related to soil

429 remediation and may have potential application to relieve soil sickness under peanut

430 monocropping.

431 Function prediction analyses showed significant changes in bacterial community functions

432 at different pathway levels with long-term peanut monocropping. The analyses at KEGG levels 2

433 and 3 and $\mathrm{KO}$ level all showed similar clustering to that of bacterial community structure.

434 Samples from short-term monocropping plots were grouped into one class, and samples from

435 long-term plots were grouped into another. Comparing analyses of the abundance indicated that

436 many detected KEGG pathways at KEGG levels 2 or 3 had obvious changes with long-term

437 monocropping. Most had a decreasing trend with long-term peanut monocropping, and only a 
438 few showed an increase in abundance. Combined with the community structure variation

439 analyses, both the bacterial community structure and function presented significant changes with

440 long-term monocropping, and their simplification could be the main cause of soil sickness.

441 In conclusion, through tracking the detailed feedback responses of soil bacteria to long-term

442 monocropping of four different peanut varieties, we provided field-based evidence that long-term

443 monocropping could result in a general loss in bacterial diversity and remarkable changes in

444 bacterial abundance and compositions as well as functions. Combined with our previous study

445 (Chen et al., 2014), analyses in this study suggested that bacterial communities were obviously

446 influenced by the monocropping period, but less influenced by peanut variety and growth stage.

447 Additionally, soil $\mathrm{pH}$ and contents of available $\mathrm{P}$ and $\mathrm{K}$ in soil also obviously changed with long-

term monocropping of peanut. Some bacterial taxa, with increased abundance have functions of

promoting plant growth or degrading potential soil allelochemicals, should be closely related to

soil remediation and may have potential application to relieve peanut soil sickness. A decrease in

diversity and abundance of bacterial communities, especially beneficial communities, and

simplification of bacterial community function with long-term peanut monocropping could be

the main cause of peanut soil sickness. In the future, we will investigate dynamics of functional

genes with long-term peanut monocropping using metagenomics. These studies will improve our

understanding of the mechanism underlying peanut soil sickness.

456

457

458

\section{REFERENCES}

Ajithkumar VP, Ajithkumar B, Iriye R, Takamizawa, K. 2003. Degradation of sodium dodecyl sulfate by Bacillus funiculus NAF001 isolated from Activated Sludge. Japanese Journal of Water Treatment 
Biology 39(3): 153-156 DOI org/10.2521/jswtb.39.153.

Asaduzzaman M, Asao T. 2012. Autotoxicity in beans and their allelochemicals. Scientia Horticulturae 134: 26-31 DOI org/10.1016/j.scienta.2011.11.035.

Bever JD, Platt TG, Morton ER. 2012. Microbial population and community dynamics on plant roots and their feedbacks on plant communities. Annual Review of Microbiology 66:265-283 DOI 10.1146/annurev-micro-092611-150107.

Blagodatskaya E, Kuzyakov Y. 2013. Active microorganisms in soil: critical review of estimation criteria and approaches. Soil Biology and Biochemistry 67:192-211 DOI org/10.1016/j.soilbio.2013.08.024.

Buttimer C, McAuliffe O, Ross RP, Hill C, O’Mahony J, Coffey A. 2017. Bacteriophages and bacterial plant diseases. Frontiers in Microbiology 8: 34 DOI org/10.3389/fmicb.2017.00034.

Caporaso JG, Kuczynski J, Stombaugh J, Bittinger K, Bushman FD, Costello EK, Fierer N, Peña AG, Goodrich JK, Gordon JI, Huttley GA, Kelley ST......Knight R. 2010. QIIME allows analysis of highthroughput community sequencing data. Nature Methods 7(5):335-336 DOI 10.1038/nmeth.f.303.

Chaparro JM, Sheflin AM, Manter DK, Vivanco JM. 2012. Manipulating the soil microbiome to increase soil health and plant fertility. Biology and Fertility of Soils 48(5):489-499 DOI org/10.1007/s00374-0120691-4.

Chen M, Chi X, Pan L, Chen N, Wang T, Wang M, Yang Z, Yu S. 2016. Research progress of soil microenvironment and peanut continuous cropping obstacle mechanism. Journal Anhui Agricultural Science 44(33):33-35 DOI 10.13989/j.cnki.0517-6611.

Chen M, Li X, Yang Q, Chi X, Pan L, Chen N, Yang Z, Wang T, Wang M, Yu S. 2012. Soil eukaryotic microorganism succession as affected by continuous cropping of peanut-pathogenic and beneficial fungi were selected. Plos One 7(7):e40659 DOI 10.1371/journal.pone.0040659. 
481

482

483

484

485

486

487

488

489

490

491

492

493

494

495

496

497

498

499

500

501

502

503

504

Chen M, Li X, Yang Q, Chi X, Pan L, Chen N, Yang Z, Wang T, Wang M, Yu S. 2014. Dynamic succession of soil bacterial community during continuous cropping of peanut (Arachis hypogaea L.). Plos One 9(7): e101355 DOI 10.1371/journal.pone.0101355.

Compant S, Clément C, Sessitsch A. 2010. Plant growth-promoting bacteria in the rhizo-and endosphere of plants: their role, colonization, mechanisms involved and prospects for utilization. Soil Biology and Biochemistry 42(5):669-678 DOI org/10.1016/j.soilbio.2009.11.024.

Cook RJ. 2006. Toward cropping systems that enhance productivity and sustainability. Proceedings of the National Academy of Sciences 103:18389-18394 DOI 10.1073/pnas. 0605946103

Doornbos RF, van Loon LC, Bakker PA. 2012. Impact of root exudates and plant defense signaling on bacterial communities in the rhizosphere. A review. Agronomy for Sustainable Development 32(1):227243 DOI org/10.1007/s13593-011-0028-y.

Edgar RC. 2013. UPARSE: highly accurate OTU sequences from microbial amplicon reads. Nature Methods 10(10):996-998 DOI 10.1038/nmeth.2604.

Fida TT, Palamuru S, Pandey G, Spain JC. 2014. Aerobic biodegradation of 2, 4-dinitroanisole by Nocardioides sp. strain JS1661. Applied and Environmental Microbiology 80(24):7725-7731 DOI 10.1128/AEM.02752-14.

Gentry LF, Ruffo ML, Below FE. 2013. Identifying factors controlling the continuous corn yield penalty. Agronomy Journal 105(2):295-303 DOI 10.2134/agronj2012.0246.

Gomaa EZ. 2012. Chitinase production by Bacillus thuringiensis and Bacillus licheniformis: their potential in antifungal biocontrol. The Journal of Microbiology 50(1):103-111 DOI org/10.1007/s12275-012-1343-y.

Gonzalez-Bashan LE, Lebsky VK, Hernandez JP, Bustillos JJ, Bashan Y. 2000. Changes in the metabolism of the microalga Chlorella vulgaris when coimmobilized in alginate with the nitrogen-fixing Phyllobacterium myrsinacearum. Canadian journal of microbiology 46(7):653-659 DOI $\operatorname{org} / 10.1139 /$ w00-041.

Peer] reviewing PDF | (2019:12:43788:2:0:NEW 10 Mar 2020) 
505

506

507

508

509

510

511

512

513

514

515

516

517

518

519

520

521

522

523

524

525

526

527

528

Ho A, Angel R, Veraart AJ, Daebeler A, Jia Z, Kim SY, Kerckhof FM, Boon N, Bodelier PLE. 2016. Biotic interactions in microbial communities as modulators of biogeochemical processes: methanotrophy as a model system. Frontiers in Microbiology 7:1285 DOI 10.3389/fmicb.

Huang LF, Song LX, Xia XJ, Mao WH, Shi K, Zhou YH, Yu JQ. 2013. Plant-soil feedbacks and soil sickness: from mechanisms to application in agriculture. Journal of Chemical Ecology 39(2):232-242 DOI org/10.1007/s10886-013-0244-9.

Ikunaga Y, Sato I, Grond S, Numaziri N, Yoshida S, Yamaya H, Hiradate S, Hasegawa M, Toshima H, Koitabashi M, Ito M, Karlovsky P, Tsushima S. 2011. Nocardioides sp. strain WSN05-2, isolated from a wheat field, degrades deoxynivalenol, producing the novel intermediate 3-epi-deoxynivalenol. Applied microbiology and biotechnology 89(2):419-427 DOI org/10.1007/s00253-010-2857-z.

Jiao K, Chen M, Pan L, Chi X, Chen N, Wang T, Wang M, Yang Z, Yu S. 2015. Effect of long term continuous cropping on the growth, yield and quality of different peanut varieties. Chinese Agricultural Science Bulletin 31(15):44-51. doi: 10.11924/j.issn.1000-6850.casb14120010.

Lee S, Ka JO, Song HG. 2012. Growth promotion of Xanthium italicum by application of rhizobacterial isolates of Bacillus aryabhattai in microcosm soil. The Journal of Microbiology 50(1):45-49 DOI org/10.1007/s12275-012-1415-z.

Li P, Dai C, Wang X, Zhang T, Chen Y. 2012. Variation of soil enzyme activities and microbial community structure in peanut monocropping system in subtropical China. African Journal of Agricultural Research 7(12):1870-1879 DOI 10.5897/AJAR11.1713.

Li XG, Ding CF, Hua K, Zhang TL, Zhang YN, Zhao L, Yang Y, Liu JG, Wang XX. 2014a. Soil sickness of peanuts is attributable to modifications in soil microbes induced by peanut root exudates rather than to direct allelopathy. Soil Biology and Biochemistry 78:149-159 DOI org/10.1016/j.soilbio.2014.07.019.

Li XG, Ding CF, Liu JG, Zhang TL, Wang XX. 2015. Evident response of the soil nematode community to consecutive peanut monoculturing. Agronomy Journal 107(1):195-203 DOI 10.2134/agronj14.0257. 
529 Li XG, Ding CF, Zhang TL, Wang XX. 2014b. Fungal pathogen accumulation at the expense of plant-

530

531

532

533

534

535

536

537

538

539

540

541

542

543

544

545

546

547

548

549

550

551

552

553

beneficial fungi as a consequence of consecutive peanut monoculturing. Soil Biology and Biochemistry

72:11-18 DOI org/10.1016/j.soilbio.2014.01.019.

Liu W, Wang Q, Wang B, Wang X, Franks AE, Teng Y, Li Z, Luo Y. 2015. Changes in the abundance and structure of bacterial communities under long-term fertilization treatments in a peanut monocropping system. Plant and Soil 395(1-2):415-427 DOI 10.1007/s11104-015-2569-3.

Liu X, Li Y, Han B, Zhang Q, Zhou K, Zhang X, Hashemi M. 2012. Yield response of continuous soybean to one-season crop disturbance in a previous continuous soybean field in Northeast China. Field Crops Research 138:52-56 DOI 10.1016/j.fcr.2012.09.012.

Lu RK. 1999. Analytical methods of soil agricultural chemistry. China Agriculture Science and Technology Press, Beijing (in Chinese)

Mellbye BL, Spieck E, Bottomley PJ, Sayavedra-Soto LA. 2017. Acyl-homoserine lactone production in nitrifying bacteria of the genera Nitrosospira, Nitrobacter, and Nitrospira identified via a survey of putative quorum-sensing genes. Applied and Environmental Microbiology 83(22): e01540-17 DOI 10.1128/AEM.01540-17.

Quast C, Pruesse E, Yilmaz P, Gerken J, Schweer T, Yarza P, Peplies J, Glöckner FO. 2013. The SILVA ribosomal RNA gene database project: improved data processing and web-based tools. Nucleic Acids Research 41:590-596 DOI 10.1093/nar/gks1219.

Ramesh A, Sharma SK, Sharma MP, Yadav N, Joshi OP. 2014. Inoculation of zinc solubilizing Bacillus aryabhattai strains for improved growth, mobilization and biofortification of zinc in soybean and wheat cultivated in Vertisols of central India. Applied Soil Ecology 73:87-96 DOI org/10.1016/j.apsoil.2013.08.009.

Santoyo G, Orozco-Mosqueda MDC, Govindappa M. 2012. Mechanisms of biocontrol and plant growthpromoting activity in soil bacterial species of Bacillus and Pseudomonas: a review. Biocontrol Science and Technology 22(8):855-872 DOI org/10.1080/09583157.2012.694413.

Peer] reviewing PDF | (2019:12:43788:2:0:NEW 10 Mar 2020) 
554

555

556

557

558

559

560

561

562

563

564

565

566

567

568

569

570

571

572

573

574

575

Sharma SK, Ramesh A, Sharma MP, Joshi OP, Govaerts B, Steenwerth KL, Karlen DL. 2010. Microbial community structure and diversity as indicators for evaluating soil quality. Biodiversity, Biofuels, Agroforestry and Conservation Agriculture 317-358 DOI org/10.1007/978-90-481-9513-8_11.

Takagi K, Fujii K, Yamazaki K, Harada N, Iwasaki A. 2012. Biodegradation of melamine and its hydroxy derivatives by a bacterial consortium containing a novel Nocardioides species. Applied Microbiology and Biotechnology 94(6):1647-1656 DOI org/10.1007/s00253-011-3673-9.

Uria AR, Piel J, Wakimoto T. 2018. Biosynthetic Insights of Calyculin-and Misakinolide-Type Compounds in "Candidatus Entotheonella sp." Methods in enzymology Academic Press 604: 287-330 DOI org/10.1016/bs.mie.2018.02.017.

van der Heijden MG. Wagg C. 2013. Soil microbial diversity and agro-ecosystem functioning. Plant and Soil 363(1-2):1-5 DOI org/10.1007/s11104-012-1321-5.

Van der Putten WH, Bardgett RD, Bever JD, Bezemer TM, Casper BB, Fukami T, Kardol P, Klironomos JN, Kulmatiski A, Schweitzer JA, Suding KN, Van de Voorde TFJ, Wardle DA. 2013. Plant-soil feedbacks: the past, the present and future challenges. Journal of Ecology 101(2):265-276 DOI $10.1111 / 1365-2745.12054$.

Vukicevich E, Lowery T, Bowen P, Úrbez-Torres JR, Hart M. 2016. Cover crops to increase soil microbial diversity and mitigate decline in perennial agriculture. A review. Agronomy for Sustainable Development 36(3):48 DOI org/10.1007/s13593-016-0385-7.

Wang HW, Sun K, Guan YX, Qiu MH, Zhang L, Dai CC. 2019. Fungal endophyte Phomopsis liquidambari biodegrades soil resveratrol: a potential allelochemical in peanut monocropping systems. Journal of the Science of Food and Agriculture 99(13):5899-5909 DOI org/10.1002/jsfa.9865.

Wang MZ, Chen XN. 2005. Obstacle and countermeasure of sustainable high yield for peanut in low-hilly red 
576

577

578

579

580

581

582

583

584

585

586

587

588

589

590

591

592

soil region. Journal of Peanut Science 34(2):17-22 DOI 10.14001/j.issn.1002-4093.2005.02.003.

Wu L, Wang J, Huang W, Wu H, Chen J, Yang Y, Zhang Z, Lin W. 2015. Plant-microbe rhizosphere interactions mediated by Rehmannia glutinosa root exudates under consecutive monoculture. Scientific Reports 5:15871 DOI 10.1038/srep15871.

Xiong W, Zhao Q, Zhao J, Xun W, Li R, Zhang R, Wu H, Shen Q. 2015. Different continuous cropping spans significantly affect microbial community membership and structure in a vanilla-grown soil as revealed by deep pyrosequencing. Microbial ecology 70(1):209-218 DOI 10.1007/s00248-014-0516-0.

Yang G, Ma K, Lu F, Wei CH, Dai XH. 2015. Effect of Continuous Cropping of Potato on Allelochemicals and Soil Microbial Community. Journal of Ecology and Rural Environment 5:15 DOI 10.11934 /j.issn.1673-4831. 2015.05. 015.

Zhou X, Liu J, Wu F. 2017. Soil microbial communities in cucumber monoculture and rotation systems and their feedback effects on cucumber seedling growth. Plant and Soil 415:507-520 DOI 10.1007/s11104017-3181-5.

Zhou X, Wang Z, Jia H, Li L, Wu F. 2018. Continuously monocropped Jerusalem artichoke changed soil bacterial community composition and ammonia-oxidizing and denitrifying bacteria abundances. Frontiers in Microbiology 9:705 DOI org/10.3389/fmicb.2018.00705. 
Figure 1

Overall abundance distribution of bacteria at the (A) class and (B) genus levels from soil that was monocropped with peanut. 


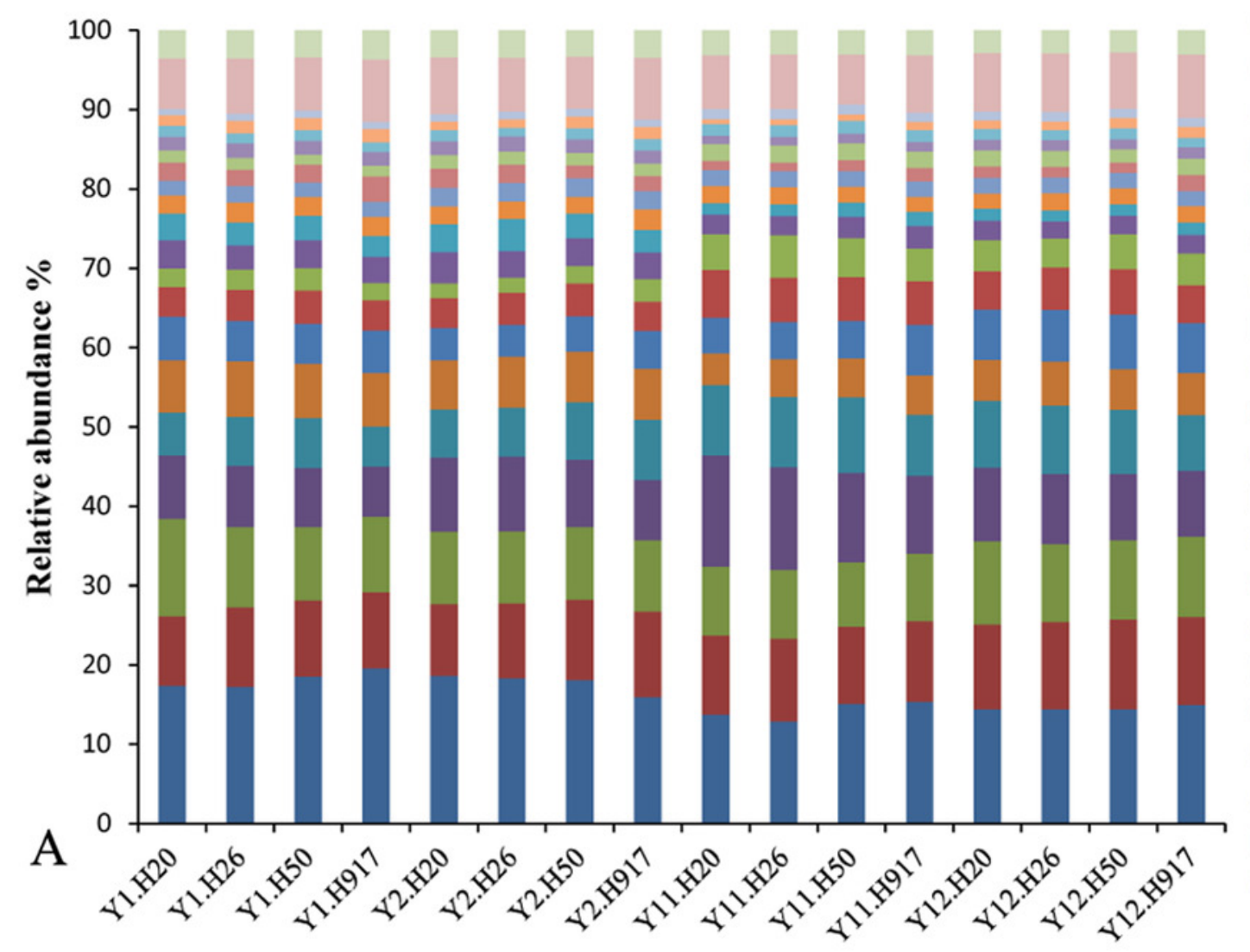

unclassified

others

= TK10

- Sphingobacteriia

n Thermomicrobia

- Holophagae

- KD4-96

Anaerolineae

n Acidimicrobiia

n Planctomycetacia

MB-A2-108

- Nitrospira

nacilli

घ Gemmatimonadetes

- Gammaproteobacteria

- Deltaproteobacteria

- Actinobacteria

- Thermoleophilia

- Betaproteobacteria

- Alphaproteobacteria

- Acidobacteria

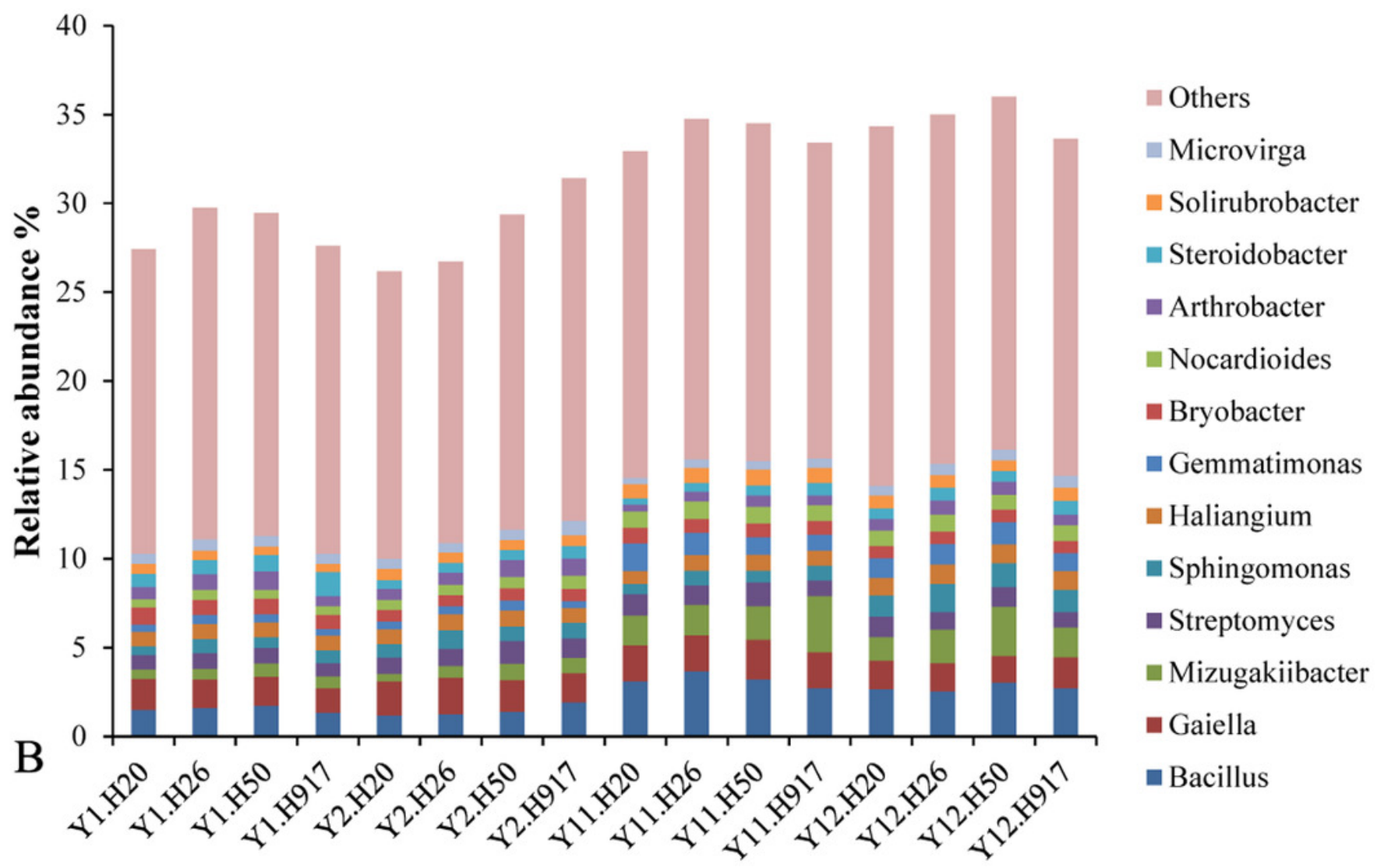


Figure 2

The UPGMA tree showing the clusters for bacterial communities in short- and long-term monocropped soils based on the unweighted UniFrac value.
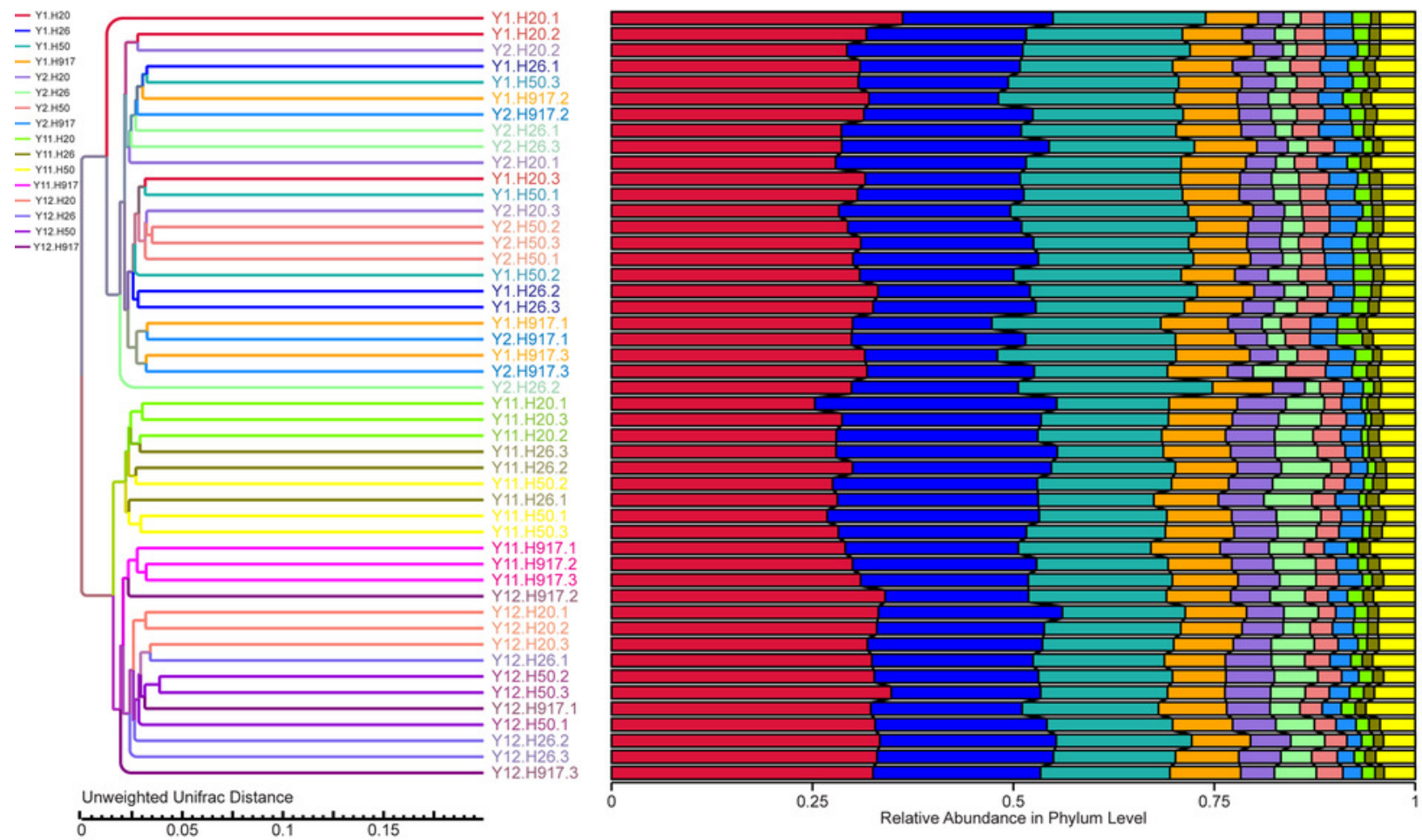

Proteobacteria

Actinobacteria

Acidobacteria

Chloroflexi

Gemmatimonadetes

$\square$ Firmicutes

Planctomycetes

Nitrospirae

$\square$ Bacteroidetes

Thermomicrobia

others

Relative Abundance in Phylum Level 
Figure 3

Principal component analysis (PCA) of bacterial distributions in short- and long-term monocropped peanut soils for different peanut varieties.

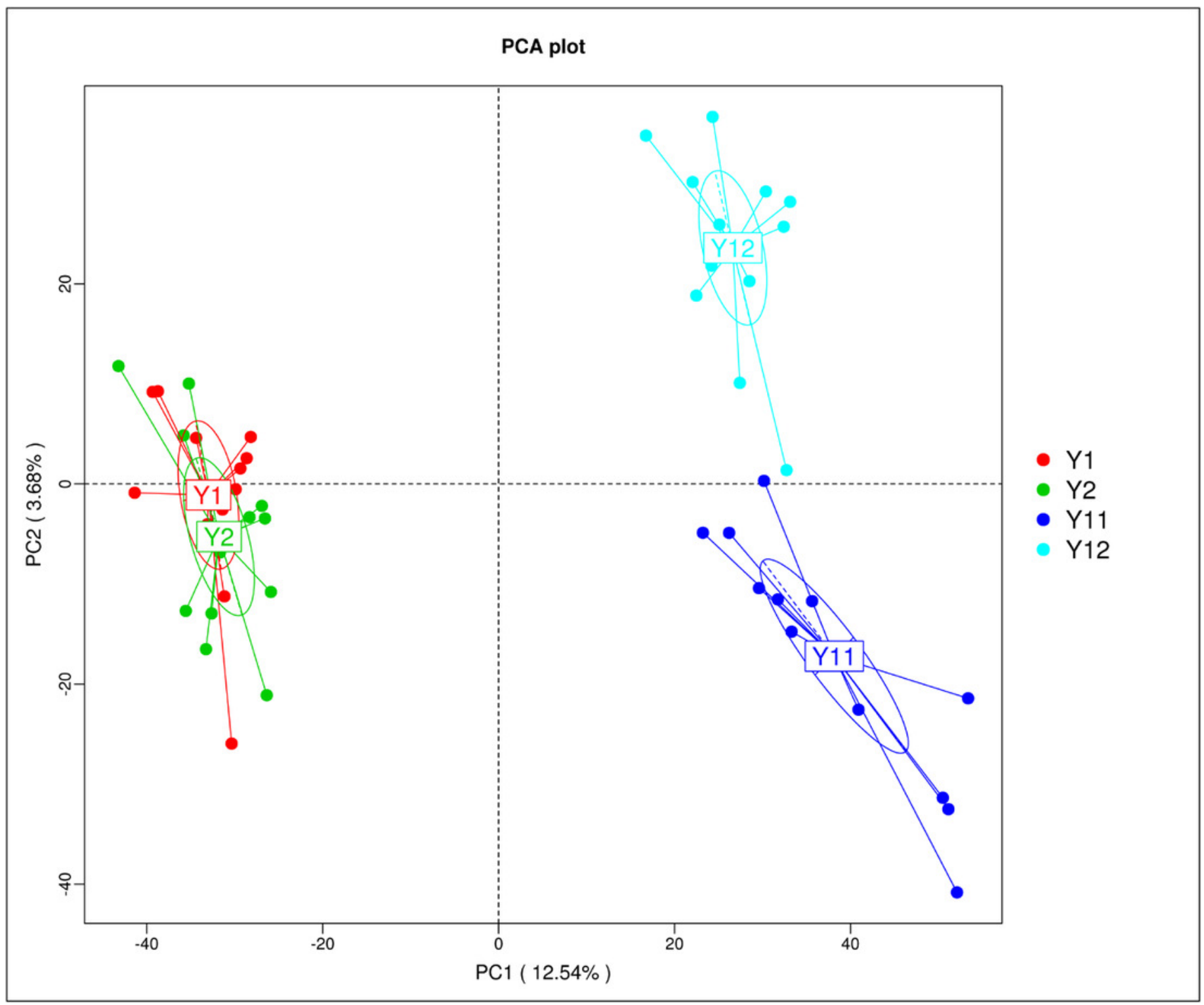


Figure 4

Relative abundance of significantly changed taxa at phylum level in plots that were monocropped with peanuts for different periods. 


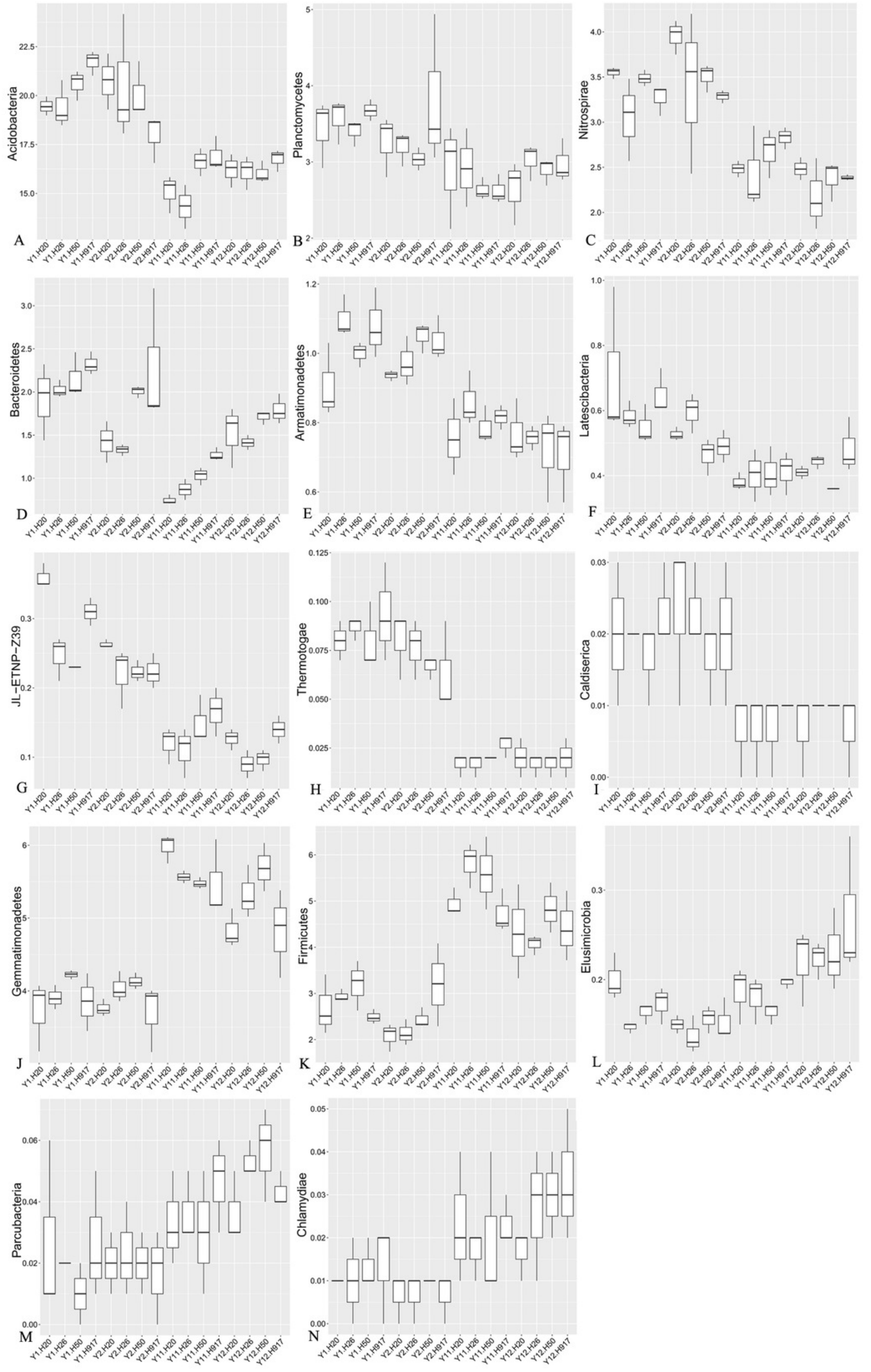


Figure 5

Heatmap presenting the distribution of the top 50 taxa at genus level in 16 monocropped peanut plots. 


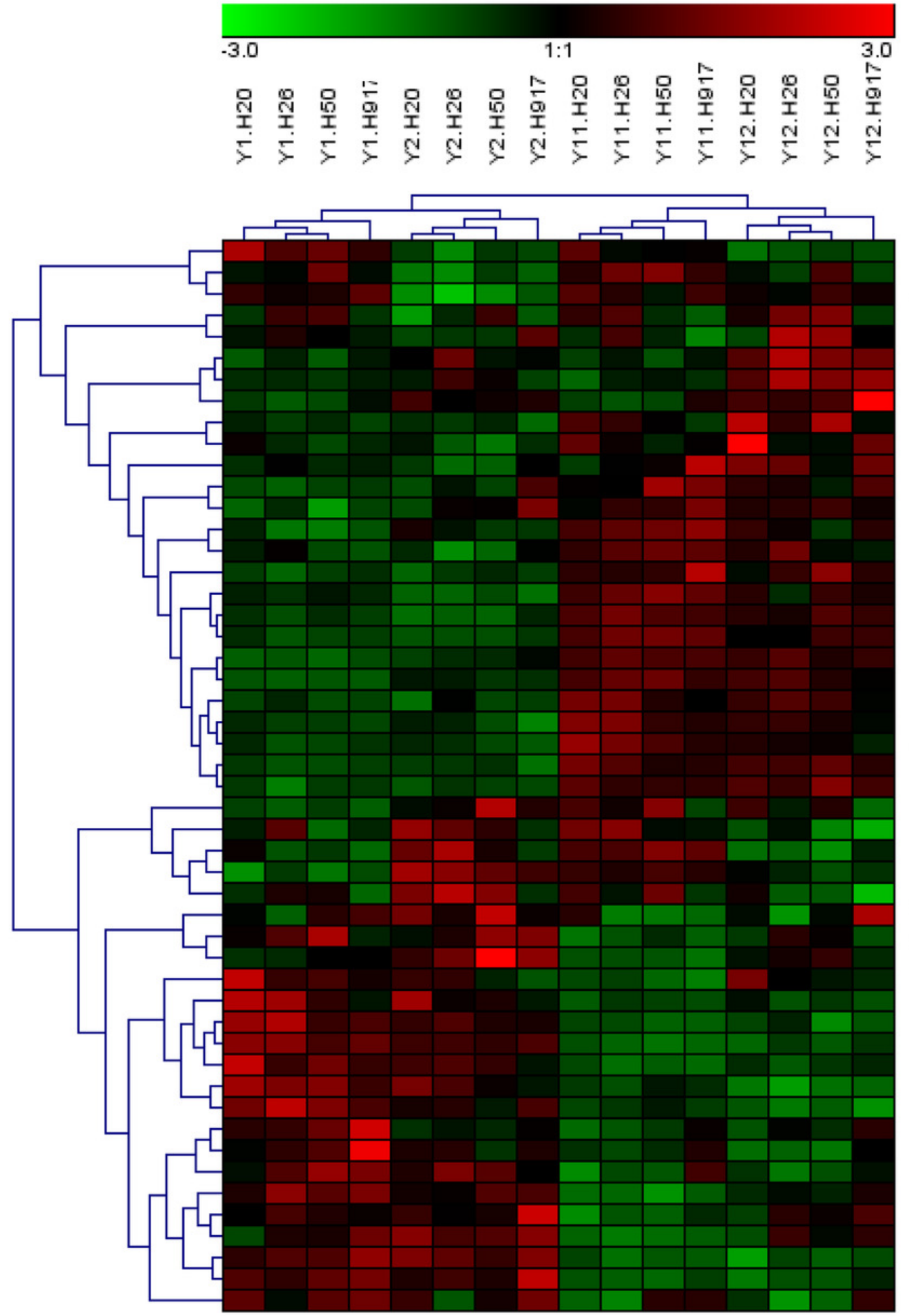

Bryobacter

Paenibacillus

Acidibacter

Bradyrhizobium

Gemmata

Sphingomonas

Haliangium

Pseudomonas

Burkholderia

Phenylobacterium

Microlunatus

Luedemannella

Mycobacterium

Solirubrobacter

Blastococcus

Mizugakiibacter

Tumebacillus

Bacillus

Sporosarcina

Nocardioides

Marmoricola

Reyranella

Candidatus Solibacter

Jatrophihabitans

Gemmatimonas

Pseudolabrys

Streptomyces

Patulibacter

Gaiella

Iamia

Kribbella

Variibacter

Arthrobacter

Novosphingobium

Cupriavidus

Nitrosospira

Piscinibacter

Ramlibacter

Lysobacter

Candidatus Entotheonella

Skermanella

Steroidobacter

Roseiflexus

Nordella

Flavisolibacter

Mic rovirga

Opitutus

Pirellula

Planctomyces

Pedomicrobium 
Figure 6

Relative abundance of significantly changed taxa at species level in plots that were monocropped with peanuts for different periods. 

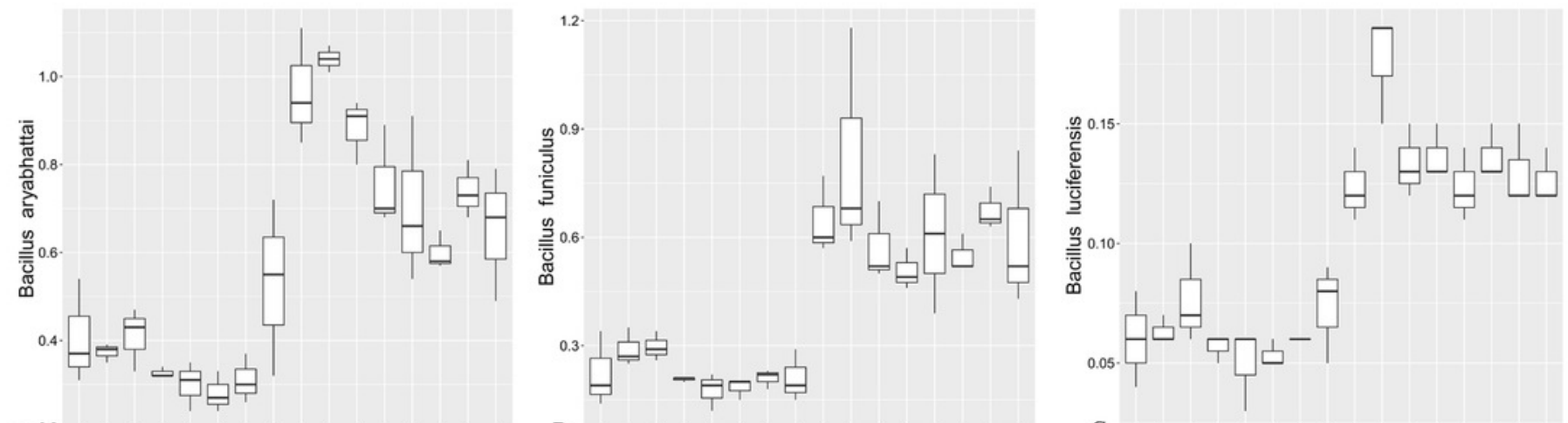

$A=0.2 .40$
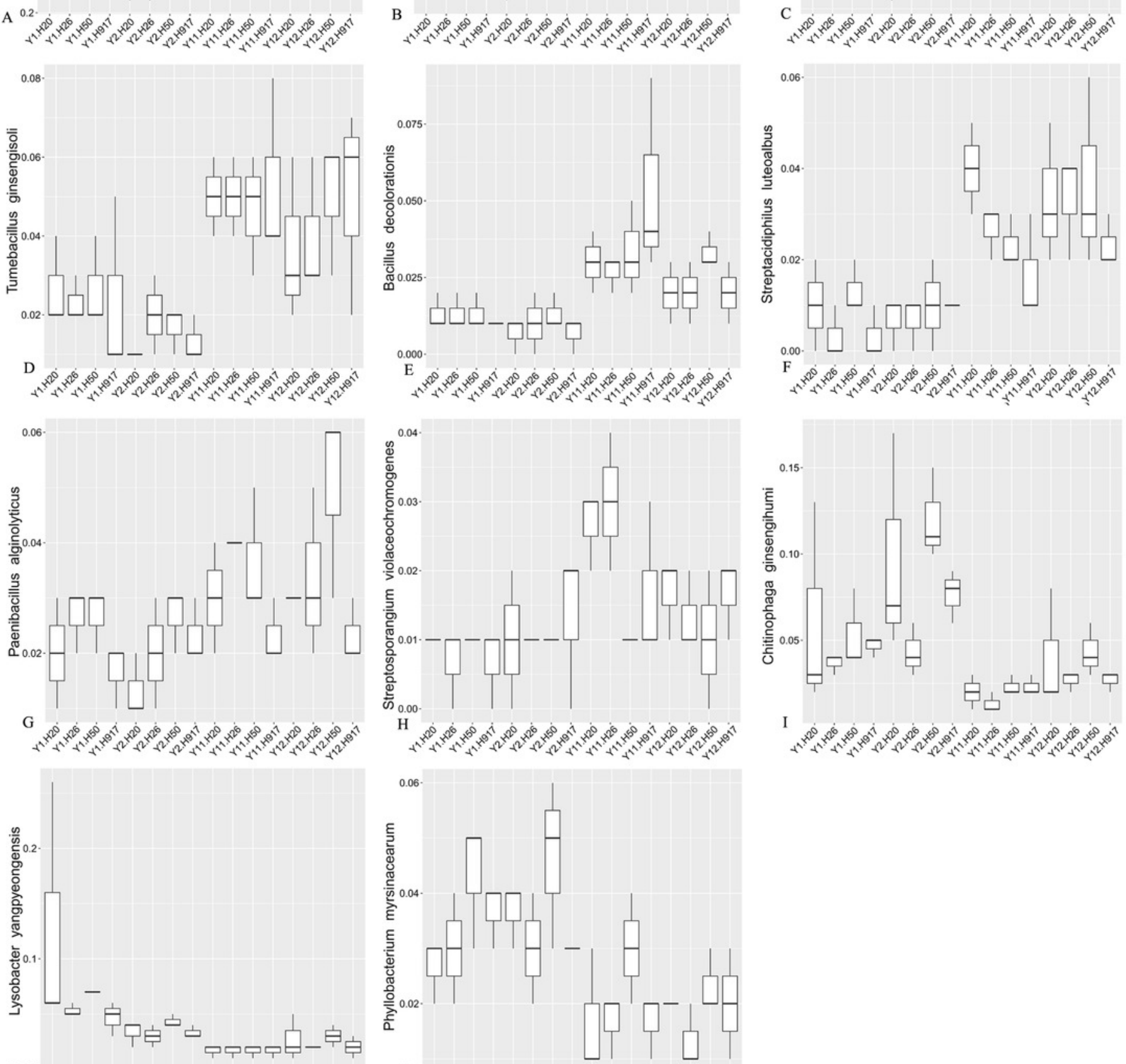

$\mathrm{J} 0.0 .000$

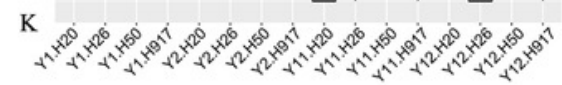


Figure 7

Principal component analysis (PCA) of predicted bacterial functions at KEGG level 2 in short- and long-term monocropped peanut soils for different peanut varieties.

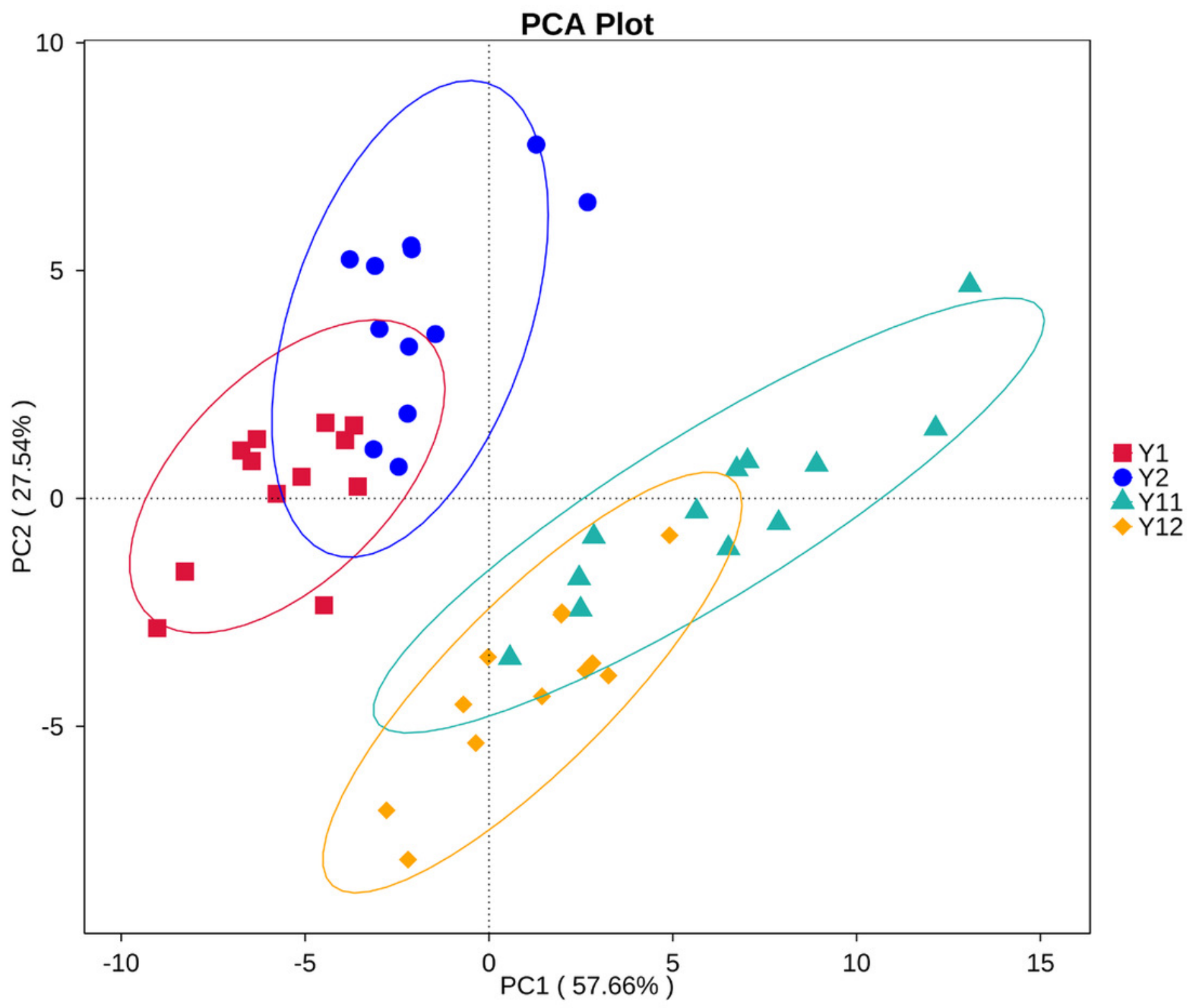




\section{Table $\mathbf{1}$ (on next page)}

Overview of soil samples obtained from plots that were monocropped for different periods.

The table shows the number of quality sequences and the indexes for $\alpha$-diversity. Operational taxonomic units (OTUs) are defined at $97 \%$ sequence similarity. 
1 Table 1. Overview of soil samples obtained from plots that were monocropped for different periods. The table 2 shows the number of quality sequences and the indexes for $\alpha$-diversity. Operational taxonomic units 3 (OTUs) are defined at 97\% sequence similarity.

4

\begin{tabular}{|c|c|c|c|c|c|c|c|}
\hline \multirow{2}{*}{$\begin{array}{l}\text { Sample } \\
\text { name }\end{array}$} & \multirow{2}{*}{$\begin{array}{c}\text { Monocropping } \\
\text { years }\end{array}$} & \multirow{2}{*}{$\begin{array}{c}\text { peanut } \\
\text { varieties }\end{array}$} & \multirow{2}{*}{$\begin{array}{l}\text { Sequenced } \\
\text { library No. }\end{array}$} & \multirow{2}{*}{$\begin{array}{c}\text { Total } \\
\text { filtered } \\
\text { quality } \\
\text { sequences } \\
\end{array}$} & \multicolumn{2}{|c|}{ Richness estimates } & \multirow{2}{*}{$\begin{array}{l}\begin{array}{l}\text { Diversity } \\
\text { estimates }\end{array} \\
\text { Shannon }\end{array}$} \\
\hline & & & & & OTUs & Chao1 & \\
\hline Y1.H20 & 1 & Huayu 20 & 3 & 211427 & $5132 \pm 327$ & $4296 \pm 727$ & $9.92 \pm 0.22$ \\
\hline Y1.H26 & 1 & Huayu 26 & 3 & 201027 & $5262 \pm 105$ & $4287 \pm 118$ & $10.10 \pm 0.06$ \\
\hline Y1.H50 & 1 & Huayu 50 & 3 & 217300 & $5187 \pm 66$ & $4189 \pm 86$ & $10.02 \pm 0.04$ \\
\hline Y1.H917 & 1 & Huayu 917 & 3 & 209336 & $5212 \pm 33$ & $4439 \pm 432$ & $10.01 \pm 0.05$ \\
\hline $\mathrm{Y} 2 . \mathrm{H} 20$ & 2 & Huayu 20 & 3 & 207920 & $5074 \pm 69$ & $4303 \pm 444$ & $9.93 \pm 0.04$ \\
\hline Y2.H26 & 2 & Huayu 26 & 3 & 197796 & $5083 \pm 139$ & $4075 \pm 166$ & $9.93 \pm 0.11$ \\
\hline Y2.H50 & 2 & Huayu 50 & 3 & 220390 & $5118 \pm 23$ & $4445 \pm 274$ & $9.99 \pm 0.03$ \\
\hline Y2.H917 & 2 & Huayu 917 & 3 & 227617 & $5462 \pm 176$ & $4839 \pm 517$ & $10.14 \pm 0.08$ \\
\hline Y11.H20 & 11 & Huayu 20 & 3 & 219846 & $4619 \pm 181$ & $3756 \pm 212$ & $9.70 \pm 0.15$ \\
\hline Y11.H26 & 11 & Huayu 26 & 3 & 196398 & $4778 \pm 71$ & $3852 \pm 115$ & $9.83 \pm 0.06$ \\
\hline Y11.H50 & 11 & Huayu 50 & 3 & 196570 & $4693 \pm 39$ & $3742 \pm 51$ & $9.83 \pm 0.00$ \\
\hline Y11.H917 & 11 & Huayu 917 & 3 & 229162 & $5064 \pm 67$ & $4428 \pm 369$ & $9.90 \pm 0.05$ \\
\hline Y12.H20 & 12 & Huayu 20 & 3 & 210578 & $5036 \pm 62$ & $4056 \pm 68$ & $9.93 \pm 0.05$ \\
\hline Y12.H26 & 12 & Huayu 26 & 3 & 212672 & $5120 \pm 56$ & $4294 \pm 363$ & $9.96 \pm 0.02$ \\
\hline Y12.H50 & 12 & Huayu 50 & 3 & 199615 & $4971 \pm 63$ & $4209 \pm 321$ & $9.91 \pm 0.07$ \\
\hline Y12.H917 & 12 & Huayu 917 & 3 & 197203 & $5049 \pm 233$ & $4017 \pm 328$ & $9.97 \pm 0.07$ \\
\hline
\end{tabular}

